\title{
Health literacy and health outcomes in China's floating population: mediating effects of health services
}

Haiyan Yu ( $\square$ yuhaiyanfei@163.com )

Wenzhou Medical University

Wei-ling Wu

Wenzhou Medical University

Lei Wu

Shaoxing University College of Business and Management

lin-wei Yu

Wenzhou Medical University

Research article

Keywords: floating population, health literacy, health outcomes, health services

Posted Date: June 16th, 2020

DOI: https://doi.org/10.21203/rs.3.rs-32594/v1

License: (a) (i) This work is licensed under a Creative Commons Attribution 4.0 International License.

Read Full License

Version of Record: A version of this preprint was published at BMC Public Health on April 8th, 2021. See the published version at https://doi.org/10.1186/s12889-021-10662-7. 


\title{
Health literacy and health outcomes in China's floating population: mediating effects of
}

\section{health services}

Hai-YanYu ${ }^{+} * 1$,Wei-Ling $\mathrm{Wu}^{1}$,Lin-wei $\mathrm{Yu}^{1}$,Lei Wu ${ }^{2}$

\begin{abstract}
Background: Health literacy is an important behavioral factor for promoting health and disease prevention. This study aimed to examine whether health literacy affected health outcomes in China's floating population and whether health service utilization had a mediating effect between health literacy and health outcomes. Method: A cross-sectional study was carried out in Zhejiang Province, China, in November and December 2019. Self-reported questionnaires were used for data collection, which included sociodemographic characteristics, health literacy, health outcomes, and health service utilization. On the basis of reliability testing, confirmatory factor analysis was used to test questionnaire validity. Descriptive statistics were used to understand the demographic characteristics of the floating population, and structural equation modeling was used for the mediation test to check whether health service utilization had a mediating effect between health literacy and health outcomes. Results: There were positive correlations between health literacy, health service utilization, and health outcomes; correlation coefficients ranged from 0.165 to 0.944 . Mediation analysis showed that health service utilization had partial mediating effects between health literacy and health outcomes. In the relationship between health literacy and health outcomes, the indirect effects of health service utilization accounted for $6.6 \%-8.7 \%$ of the total effects. Conclusion: Health service utilization has partial mediating effects between health literacy and health outcomes. Health literacy affects the proactiveness of health service utilization in the floating population through healthcare

\footnotetext{
* Correspondence: yuhaiyanfei@163.com;

Hai-Yan Yu is the first authors

${ }^{1}$ 7B304, School of Public Health Management, Wenzhou Medical University, Chashan Town, Ouhai District, Wenzhou City, China ${ }^{2}$ Shaoxing College of Arts and Sciences, 508 Huancheng West Road, Shaoxing, China
} 
literacy and health promotion, thereby affecting health outcomes.

Keywords: floating population, health literacy, health outcomes, health services 


\section{BACKGROUND}

As a typical marker of economic development and social progress, migration brings about changes in employment, the living environment, and social networks. In the context of rapid ongoing urbanization, large-scale population migration will continue to be an important factor in China's socioeconomic development for a long time. That said, population migration requires healthy citizens, which makes health a prerequisite for the survival and development of the floating population.

China's floating population is vulnerable. A survey in Zhejiang Province found that the incidence of measles was positively correlated with the proportion of floating population in the province. The main reason for this phenomenon is the low vaccination rate among provincial floating populations [1]. Migrant health is affected by various factors, including insufficient health awareness, limited financial capacity, place of residence, and work environment [2]. One study found that self-measurement scores for the floating population were significantly lower than those for the local household population. For physical health, the low-scoring items included those for fatigue and gastrointestinal symptoms; for psychological health, they included anxiety and depression. The psychological health of floating populations typically corresponds to how well they can integrate with local residents [3].

Regarding research on migrant health outside of China, a study in the US found that the risk of cardiovascular disease was higher in the floating population than in the local population [4]. Among African immigrant youth in Canada, problems related to regional discrimination, identity, and cultural impact adversely affected psychological health [5]; yet, only $13.3 \%$ were willing to seek out psychological health services [6]. Immigrants tend to experience heavy mental burdens after settling in a new area [7], and this is certainly true of China's floating population. As such, 
it is important to focus on the health problems of this population.

Proposing that the individual is ultimately responsible for his or her health, the "Healthy China" strategy advocated increasing health literacy, shifting the focus from disease treatment to prevention, and accelerating the adoption of healthy lifestyles. The World Health Organization has defined health literacy as "the cognitive and social skills which determine the motivation and ability of individuals to gain access to, understand and use information in ways which promote and maintain good health" [8]. Studies have found that health literacy is associated with access to health services, and lower health literacy leads to adverse health behavior and outcomes $[9,10]$. One study found that increased disease-prevention literacy in a Turkish rural population was positively correlated with vaccination rates [11]. Due to low health literacy, China's floating population is at a high risk for infectious diseases; in particular, a lack of reproductive health knowledge has increased the spread of sexually transmitted diseases [12]. Many youths in the floating population have irregular diets, excess stress, and a lack of routine medical treatment. One study found a correlation between the health literacy level of Korean migrants and the risk factors for type 2 diabetes [13]. Moreover, poor health literacy and poor care were found to be correlated with HIV infection among the African American population [14]. Meanwhile, one study found that while locals and immigrants face similar mental health problems, immigrants are less likely to use mental health services [15]. Another study found that health literacy could pose a barrier to accessing health services among vulnerable populations, thereby resulting in poor health outcomes [16].

Health service utilization includes medical, healthcare, and rehabilitation service utilization. One study found that maternal health services significantly reduced mortality rates among pregnant women [17]. Healthcare systems based on place of residence and employment status 
can make it difficult for migrants to access health services [18]. Due to the restrictions of China's household registration system, the floating population has low awareness of health services [19], poor utilization of health services, and low vaccination rates compared to local populations [20]. Outside of China, health service utilization by immigrants is similarly affected by health literacy. In New York City, low mental health service utilization rates among elderly Chinese immigrants increased the risk of mental illness [21]. In Brazil, only $45.6 \%$ of immigrant subjects used some form of medical service [22]. In light of such findings, there is a need to examine how to improve health literacy among floating populations to promote health service utilization.

In summary, based on the abovementioned studies of both Chinese and international migrants, we should focus on three dimensions of health promotion to improve the health of the floating population-namely, health awareness, health behavior, and supportive environments. Health awareness and health behavior are part of health literacy while a supportive environment is related to providing various external health services. An individual's health literacy must merge with a supportive external environment to ultimately improve health outcomes. This study aimed

to examine the relationship between health literacy and health outcomes in the floating population and determine whether health service utilization has a mediating effect in this relationship.

\section{METHOD}

The data used in this study were obtained from Zhejiang Province, China. Zhejiang is located at the southern part of the Yangtze River Delta and includes two subprovincial cities, nine prefecture-level cities, 35 counties, 21 county-level cities, and 34 urban districts. The "2018 China Floating Population Development Report" indicated that China's floating population was 
241 million people at the end of 2017 [23]. In terms of cross-provincial migrant population, Guangdong, Zhejiang, Shanghai, and Beijing accounted for 32.25\%, 22.57\%, 12.62\%, and $8.01 \%$, respectively, of the floating population. After the floating population in Zhejiang had exceeded that of Shanghai and Jiangsu in 1997 and 2000, respectively, Zhejiang had the second-largest floating population in China for 19 continuous years. This, along with the province's level of economic development, is why Zhejiang was selected for this study. For the study sites, we selected Hangzhou, Ningbo, and Wenzhou, which are representative cities with internal migrant aggregation in China. Before conducting the survey, a preliminary test was carried out in which 150 questionnaires were distributed and 148 were collected (recovery rate: 98\%). Cronbach's alpha tests and confirmatory factor analysis were conducted on the questionnaire before revision. The revised questionnaire was then used to carry out a large-scale survey in November 2019.

The survey period was November to December 2019. The 2017 annual floating population data for Zhejiang Province were used as the sampling frame basis. A stratified, multistage, scale-proportion probability proportional to size (PPS) method was used for sampling. The top three cities with the largest floating populations in Zhejiang were selected for the survey (i.e., Hangzhou, Ningbo, and Wenzhou). Three sampling sites were randomly selected for each city, and three communities were randomly selected in the sampling site. Then, the researchers selected $20-40$ people from the floating population in each selected community according to gender, age, and migration time. The inclusion criteria included people who had been in Zhejiang for at least one year before the survey, did not have a registered household address in the region (county, city), and were over age 15 in November 2019. The exclusion criteria included students and transient populations at train stations, harbors, airports, hotels, and hospitals. A total of 670 
questionnaires were distributed online and offline, and 657 valid questionnaires were recovered (validity rate: 98\%). Before the subject completed the self-reported questionnaire, the investigator explained the aim of study, the data collection method, and how to complete the survey. The investigators also informed subjects that participation was anonymous and voluntary.

\section{Measurements}

\section{Health gain}

The dependent variables of self-evaluated health, physiological health, and psychological health were used to examine health status in the floating population. The short-form 36 questionnaire (SF-36) from the US was used to assess self-evaluated health [24]; it mainly measures self-perceived health compared to others around the subject using a five-point Likert scale. A simplified version of the Patient Health Questionnaire (PHQ-15) was used to measure physiological health; the Chinese version has shown good validity and reliability [25]. The scale

includes headache, chest pain, and arthralgia, assessed on a five-point Likert scale. Finally, a simplified version of the Hopkins Symptoms Check List (HSCL) was used for psychological health. The scale mainly measures anxiety and depression using a five-point Likert scale; the higher the score, the poorer the psychological health [26].

\section{Health services}

The "2017 Chinese Floating Population Health and Family Planning Dynamic Monitoring Questionnaire" was used to evaluate health service access by the floating population. The questionnaire includes five content areas: (1) general status of family members, (2) movement trends and residence intention, (3) employment characteristics, (4) basic public health service utilization, and (5) social integration. This study used the basic public health service utilization scale, which includes 14 basic public health service items (i.e., health record management, health 
education, prophylactic vaccination, health management of 0-6-year-old children, health management of pregnant women, health management of elderly people, health management of chronic disease patients (hypertension, diabetes), management of severe mental illness patients, health management of tuberculosis patients, infectious diseases and public health emergency reporting and management services, traditional Chinese medicine health management, health and family planning monitoring services, free contraceptive tools, health literacy promotion). The Chinese Floating Population Health and Family Planning Dynamic Monitoring Questionnaire is divided into four dimensions of vaccination services, health examination, health education, and physical examination; five-point Likert scales were used to assess health services provided in these four dimensions.

\section{Health literacy}

The European Health Literacy Survey Questionnaire was used to investigate health literacy in the floating population. This questionnaire is multidimensional and has been used to measure health literacy in European populations [27]. The questionnaire is available in three versions. First, the HLS-EU-Q47 is based on a 4 (information processing: finding, understanding, judging, applying) $\times 3$ (health domain: health care, disease prevention, health promotion) matrix and contains 47 items. Second, the HLS-EU-Q86 adds health behavior, health status, health service utilization, community participation, sociodemographic, and socioeconomic factors and has 86 questions. Third, the HLS-EU-Q16 was formulated for rapid health evaluation. Among these

three, the HLS-EU-Q47 is the most widely used, and it has high validity and reliability in Asian contexts [28]. Therefore, the HLS-EU-Q47 questionnaire was used in this study. It includes 47 questions measuring health literacy scored using four-point Likert scales (1: extremely difficult to 4: extremely easy). The lowest possible mean score is 1 , and the highest is 4 . 


\section{Data analysis}

SPSS 22.0 was used for frequency analysis, reliability testing, and Pearson's correlation analysis. Amos 22.0 was used to establish a confirmatory factor model to provide validity, construct a standardized path test, and examine the hypothesis testing results; bootstrapping was used to test the mediating effects. In the reliability analysis, the baseline value for judging the questionnaire was determined to be 0.7 , with a value greater than 0.7 indicating that the questionnaire was feasible. Similarly, the Amos test of questionnaire validity was carried out using the model fit index. The specific criteria for assessment were $\mathrm{cmin} / \mathrm{df}<5$ and GFI, AGFI, NFI, TLI, and CFI $<0.8$, showing that the questionnaire had good validity. If a model has three questions, the constructed model is saturated and $\mathrm{df}$ is 0 , and the model fit results will not be evaluated. In subsequent hypothesis testing, the collinearity test results were first used to ensure the inflation factor of the variable did not exceed 10 to show that collinearity was absent between variables. At the same time, the common-method variance test was carried out. Harman's single-factor test was used to determine that the explanatory power of the first component in the initial eigenvalue was $42.972 \%$, which is lower than $50 \%$, showing that common-method variance was absent between variables. After that, path modeling and mediator model testing were conducted. The basis of the path modeling was the theoretical model (Figure 1). Figure 1 shows that healthcare, disease prevention, and health promotion were the independent variables; health service utilization was a mediator; and health gain was the dependent variable; these were used to construct the path model. In this study, the path significance was $p<0.05$, showing that the predicted relationship between the variables was proven. At the same time, bootstrapping was used for the mediator test to obtain the total, direct, and mediating effects of various variables. A total of 2,000 random sampling calculations were used to obtain the $95 \%$ confidence interval of 
the estimated value. If the confidence interval contains 0 , the test results are not true; otherwise, the test results are true.

\section{RESULTS}

Table 1 shows the sociodemographic characteristics of the study participants. Among the 657 subjects, there were 244 males (37.1\%) and 413 females (62.9\%). The proportion of subjects aged $<40$ years was $75.6 \%$, and $78.2 \%$ had an educational level of high school or below. The proportion of subjects with a bachelor's degree was $9.4 \%$.

Table 2 shows the reliability analysis of the scale and the variable means. Cronbach's $\alpha$ consistency coefficient was used to test internal consistency reliability, and the threshold value was 0.7. A Cronbach's $\alpha$ greater than 0.7 means the question measurement results for that dimension had consistent reliability. The results show that the reliability of various sections was greater than 0.7 , indicating that the questionnaire was reliable. The mean health literacy value showed that the health promotion literacy of the floating population was better than their healthcare literacy and disease-prevention literacy. The mean health service utilization value showed that health examination service utilization was better than other types of health services. The mean value for health outcomes showed that the overall health status of the floating population was poor, and the psychological health results were better than the physiological health results, which validates the findings of previous studies.

Table 3 shows the validity analysis results for the scale. Using Amos for structural equation modeling (SEM), the evaluation markers included CMIN/DF, NFI, IFI, TLI, CFI, RMSEA, and other fit markers. These markers were mainly used for model discrimination. Table 3 shows the specific discrimination criteria. From the confirmatory factor analyses of the three questionnaires, 
it can be seen that the model fit indices for health literacy, health service utilization, and health outcomes were within the acceptable range. Since health outcomes has only three questions and is a saturated model, a model fit test was not carried out. From this, it can be determined that the various questionnaires had good validity markers and that the questionnaires are valid.

Table 4 shows the correlation relationships between variables. The total health service utilization score and the various dimensions showed a significantly positive correlation with the total score for health literacy and various dimensions. At the same time, the total health service utilization score and the various dimensions showed a significantly positive correlation with the total score for health outcomes and various variables. Moreover, the total score for health literacy and various dimensions showed a significantly positive correlation with the total health gain score and various variables.

Tables 5 and 6 show the path analysis and mediation test. From the correlation analysis results, significant correlation relationships can be seen between various hypothesis variables. Therefore, Amos was used to construct the model.The path test table shows that all paths passed the test except for the path from disease prevention literacy to health service utilization and the path from disease prevention to health outcomes. Among the variables, healthcare had significant positive effects on health service utilization, with a coefficient of $0.227(\mathrm{p}<0.001)$. Disease prevention did not significantly affect health service utilization, with a coefficient of $0.112(\mathrm{p}=$ 0.135). Health promotion had significant positive effects on health service utilization, with a coefficient of $0.299, \mathrm{p}<0.001$. Health service utilization had significant positive effects on health outcomes, with a coefficient of $0.29(\mathrm{p}<0.001)$. Healthcare had significant positive effects on health outcomes, with a coefficient of $0.215, \mathrm{p}<0.001$. Disease prevention did not significantly predict health outcomes, with a coefficient of $-0.084(p=0.25)$. Lastly, health 
promotion had significant positive effects on health outcomes, with a coefficient of 0.248 ( $p<$ $0.001)$.

The overall model fit results were good, with $\mathrm{CMIN} / \mathrm{DF}=1.723<3$; NFL, IFI, TLI, and CFI were all greater than 0.9 , and RMSEA $=0.033<0.05$. Therefore, it can be determined that the data and the model match very well, and the model is valid.

Bootstrapping was used for the mediating effect test with 2,000 samples. The $95 \%$ confidence interval test was used to obtain the mediating effect results. The mediation test was significant for healthcare effects on health outcomes through health service utilization, with a total effect size of 0.280 ; the confidence interval [0.174-0.378] does not include 0 , indicating that the total effect is true. The direct effect size was 0.215 , and the confidence interval [0.108-0.313] does not include 0 , showing that the direct effect is true. The mediating effect size was 0.066 , and the confidence interval [0.023-0.299] does not include 0 , showing that the total effect is true, and the model is a partial mediation model. The results showed that the total effect, direct effect, and mediating effect of disease prevention on health outcomes through health service utilization were not true, indicating that health service utilization was not a mediator variable for the effects of disease prevention literacy on health outcomes. The mediation test was significant for health promotion on health outcomes through health service utilization, with a total effect size of 0.335 , and the confidence interval [0.192-0.467] does not include 0 , indicating that the total effect is true. The direct effect size was 0.248 , and the confidence interval [0.101-0.389] does not include 0 , indicating that the direct effect is true. The mediating effect size was 0.087 , and the confidence interval [0.047-0.133] does not include 0 , indicating that the total effect is true, and the model is a partial mediation model. 


\section{DISCUSSION}

This study employed SEM to examine the relationship between health literacy and health outcomes in China's floating population and simultaneously analyzed the mediating effects of health service utilization. Health literacy affected health outcomes through two dimensions - healthcare literacy and health promotion literacy_-but disease prevention did not directly affect health outcomes. The results showed that health literacy had positive effects on health outcomes, and health service utilization had partial mediating effects between health literacy and health outcomes.

Healthcare literacy directly affected health outcomes. Healthcare literacy concerns whether people are equipped with an understanding of and ability to communicate about health services and whether they possess medical skills for emergencies. The proportion of the floating population who chose to self-medicate or not adopt any measures was the highest, followed by visiting private/individual clinics or community health service stations/rural health centers. The proportion of subjects who chose to go to private/individual clinics was $1 / 5$, and the proportion of subjects who chose to go to county-level and above hospitals was the lowest [29]. Such choices are the result of interactions between subjective and objective factors and are closely associated with education level, income, occupation, health status, and disease. Self-medication was the first choice among the floating population after disease onset, which requires actively searching for treatment information. If they are unable to self-medicate, they usually suffer from a major disease and have to seek professional medical services. This requires them to be able to communicate with physicians and understand medication instructions, among other aspects of treatment. At the same time, income and social security limit the ability of the floating population to access medical services. They must therefore master certain self-treatment 
techniques, such as traditional massage, acupuncture, and cupping therapy.

Health promotion literacy directly affected health outcomes. Health promotion refers to a social behavior or strategy that uses administrative or organizational measures to coordinate various social departments, communities, families, and individuals to carry out individuals' health responsibilities to jointly maintain and promote health. In health literacy, health promotion refers to an individual's understanding of the factors affecting physical and mental health, such as governmental health policies, community facilities, social networks, work environments, and residential environments. It also refers to actively searching for relevant health education resources and making decisions about improving health. To better satisfy the health service needs of the floating population and improve their health levels, the government can rely on multicenter governance theory and social policy development directions to ask communities to construct a more comprehensive health service utilization system. Such a system would include three major providers of floating population health services: community residential (village) committees, community health service centers, and social workers/community organizations [30]. The living conditions and work environments of the floating population are generally poor, and these adverse factors have negative effects on their health. Among them, the effects of adverse work environments are more prominent. Conversely, participation in social activities, staying with family members, and socializing with local employees have important positive effects on promoting health among the floating population. Therefore, relevant departments should employ the necessary measures to improve the living conditions and work environments of the new generation of rural workers to improve their health [31].

Disease prevention did not directly affect health outcomes. Disease-prevention health literacy mainly refers to understanding the importance of health behaviors and health 
examinations. It entails understanding the effects of smoking, low exercise intensity, and excessive drinking; knowing that vaccinations and health examinations can help prevent disease; and possessing the ability to improve health behaviors. Workers should also be able to decide how to protect themselves from disease based on suggestions from friends, family, and media. In this regard, this study's results conflict with previous findings. It could be that the floating population is one that is naturally selected for health, and, generally, only healthy people can migrate. Meanwhile, most of China's floating population were originally farmers, and their low educational levels limit their understanding of nutrition, healthcare, and disease prevention. Most floating population workers do manual labor. Under profit maximization and industry competition pressure, small and medium-sized enterprises may choose to increase manufacturing speed to produce more products. They employ a piece-rate payment system to encourage workers to work longer hours. This not only affects their health but also reduces normal rest and leisure time, which includes time for exercise and attention to diet. Long working hours, poor living conditions, poor work environments, and stress related to integration cause them to lack the energy to gain disease prevention knowledge and cultivate healthy lifestyle habits.

Health service utilization directly affected health outcomes. In 2016, China proposed the "Healthy China" strategy, establishing public health as a primary objective. However, data from the "2016 China Floating Population Development Report" showed that the health of the floating population, which is an important component of the labor force, is declining [32], highlighting the need to provide medical, preventive, healthcare, and rehabilitation services for this population. Studies have shown that different household registrations affect the preventive health services provided by public health departments. To enable the floating population to obtain basic public health services, it is necessary to eliminate the separation between rural- and 
urban-registered households, increase the permanent residence rate of the floating population, promote a basic health security system for the entire country, and strengthen employment training and education levels [33]. This requires synergistic effects from top and middle governments as well as grassroots involvement. After the top government integrates health into public policies, middle governments need to expand the structural intervention of public health services for health promotion. This also requires the government to focus on providing public health services and strengthening the municipal level as a health-related decision structure [34]. The grassroots level should refer to community pharmacies in the US, which can intervene to help prevent primary diseases through smoking cessation, weight management programs, needle exchange, and vaccination services, among others [35]. Moreover, the floating population does not passively receive public services, and their participation (PPI) has become an indispensable part of healthcare, with a focus on improving their participation in public affairs [36]. Research has also found that the effects of health service announcements are better after people view health-related fictional programs [37]. Thus, public health media should not only utilize many new media channels but also find ways to attract audiences and help them gain health knowledge. Lastly, health impact evaluation is a typical task in public health services. A study in Germany found that health assessment can clarify the responsibilities of health services [38]. Therefore, assessing the impact of various types of health services on health for resource matching is a future option.

Health service utilization had partial mediating effects in the relationship between healthcare literacy and health promotion literacy and health outcomes. Health service utilization was not found to have a mediating effect in the relationship between disease prevention literacy and health outcomes. The effects of health literacy on health outcomes can be partially explained 
by health service utilization. For example, females with lower health literacy tend to use less preventive healthcare services, including flu vaccination and cervical and breast cancer screening [39]. Moreover, low health literacy is more common among elderly people [40]. A lack of health literacy also directly affects the effective utilization of health services and social welfare by chronic disease patients and is closely associated with disease management and health outcomes [41]. Therefore, good health literacy in the floating population can enable them to clearly understand, recognize, and control the relationship between their lifestyle and health status [42]. Improving health knowledge, changing unhealthy lifestyle and work habits (e.g., working overtime or working when sick), actively using various available health resources, and making a habit of regular physical exams can form a positive feedback loop of early diagnosis, treatment, and recovery. In summary, the floating population poses a top health priority, and the establishment of a national health management system should focus on family health advocacy. Regarding the fact that health service utilization does not have mediating effects in the relationship between disease prevention and health outcomes, the root cause is that disease prevention literacy did not affect health outcomes. This is because the floating population is mostly young and has poor health-risk awareness. Meanwhile, disease prevention literacy involves professional preventive medical knowledge. Due to educational limitations, it is difficult for the floating population to have such understanding. In the future, subjects can be stratified by education level to examine the effects of disease prevention literacy on health outcomes. Given the current COVID-19 pandemic, the mobility of the floating population poses a huge health risk. Hence, there is an urgent need to strengthen their health literacy regarding disease prevention to reduce the risk posed by COVID-19.

This study has some limitations. First, the health literacy scale used in this study is a typical 
scale for critical health literacy, but there is a lack of scales for functional health literacy and communicative health literacy. In the future, the All Aspects of Health Literacy Scale (AAHLS) can be used to measure health literacy in the floating population as it is suitable for evaluating functional, communicative, and critical health literacy. In addition, there is a risk of bias when a self-evaluated health literacy tool is used since widespread optimism/pessimism and memory deficiencies can affect the outcomes. Second, the cross-sectional design did not allow for causality deduction. Conducting longitudinal studies and reassessing health outcomes will help identify causality in health literacy. Moreover, the samples came from three prefecture-level cities. The educational levels of floating populations in different parts of China are different, which affects health literacy. This could have affected sample representativeness and the generalization of the results. Lastly, we did not investigate the connection between health literacy and health behavior. Examining this relationship in the future could aid in designing intervention measures at the individual level, thereby improving the health of vulnerable populations.

\section{CONCLUSION}

This study found that health service utilization had partial mediating effects in the effects of health literacy on health outcomes. Health literacy affects the proactiveness of the floating population in health service utilization through healthcare literacy and health promotion literacy, thereby affecting health outcomes. Improving health literacy in the floating population will help improve their health outcomes. At the same time, health service providers need to enhance the diversity of health services and ensure that the floating population has the necessary external conditions to improve their individual health. 


\section{Declarations}

\section{Abbreviations}

HLS: Health literacy Survey

\section{Acknowledgements}

We would like to thank all subjects who participated in the study.

\section{Authors' contributions}

HYY conducted the data analysis and drafted the preliminary manuscript. LWY participated in writing and improving the manuscript. HYY, LWY, and WLW participated in measurement and data analysis. LW conceptualized and designed the study and provided suggestions for data interpretation. HYYwas the project coordinator and participated in all of the work. All authors read and approved the final manuscript.

\section{Authors' information}

Hai-Yan Yu is the first author and corresponding author

\section{Funding}

This work was supported by the National Social Science Fund of China (no.17BZZ054). The sponsor played no part in the study design, data collection, analysis, interpretation, manuscript writing, or study approval.

\section{Availability of data and materials}


The datasets used in this study can be obtained from the corresponding author upon reasonable request.

\section{Ethics approval and consent to participate}

This study received ethical clearance from Ethics Committee of Wenzhou Medical University. Consent was directly obtained from the participants, and all participants signed an informed consent form.

\section{Consent to publication}

Not applicable.

\section{Competing interests}

The authors declare that they have no competing interests 


\section{REFERENCES}

1. Qin S, Ding Y, Yan R, He H: Measles in Zhejiang, China, 2004-2017: Population Density and Proportion of Floating Populations Effects on Measles Epidemic. Health security 2019, 17(3):193-199.

2. Fan X: Floating population health status, problems and countermeasures. Macroeconomic Management 2019(04):42-47.

3. Yu HY, Yu LW: Social integration, social support and migrants Holistic Health - An Empirical Analysis of Zhejiang. Zhejiang Social Sciences 2018(06):86-95+157-158.

4. Turkson-Ocran R-AN, Nmezi NA, Botchway MO, Szanton SL, Golden SH, Cooper LA, Commodore-Mensah Y: Comparison of Cardiovascular Disease Risk Factors Among African Immigrants and African Americans: An Analysis of the 2010 to 2016 National Health Interview Surveys. Journal of the American Heart Association 2020, 9(5):e013220-e013220.

5. Olawo O, Pilkington B, Khanlou N: Identity-Related Factors Affecting the Mental Health of African Immigrant Youth Living in Canada. International Journal of Mental Health and Addiction 2019,4:1-3.

6. Ayele SG, Thompson-Robinson M, Andrews J, Dodge Francis C: Factors Associated with Mental Health Service Utilization Among Ethiopian Immigrants and Refugees. Journal of immigrant and minority health 2020:1-8.

7. Aragona M, Salvatore MA, Mazzetti M, Burgio A, Geraci S, Baglio G: Is the mental health profile of immigrants changing? A national-level analysis based on hospital discharges in Italy. Annali di igiene: medicina preventiva e di comunita 2020, 32(2):157-165. 
8. Tolan NV: Health Literacy and the Desire to Manage One's Own Health. Clinics in laboratory medicine 2020, 40(1):1-12.

9. Haghdoost AA, Karamouzian M, Jamshidi E, Sharifi H, Rakhshani F, Mashayekhi N, Rassafiani H, Harofteh F, Shiri M, Aligol M et al: Health literacy among Iranian adults: findings from a nationwide population-based survey in 2015. Eastern Mediterranean Health Journal 2019, 25(11):828-836.

10. Michou M, Panagiotakos DB, Lionis C, Costarelli V: Socioeconomic inequalities in relation to health and nutrition literacy in Greece. International Journal of Food Sciences and Nutrition 2019, 70(8):1007-1013.

11. Guclu OA, Demirci H, Ocakoglu G, Guclu Y, Uzaslan E, Karadag M: Relationship of pneumococcal and influenza vaccination frequency with health literacy in the rural population in Turkey. Vaccine 2019, 37(44):6617-6623.

12. Shuang FX, Yu YL, Jun QW: Floating population genital tract infection / STD / AIDS prevalence and risk factors and prevention strategies. Chinese Journal of Reproduction and Contraception 2017, 37(03):250-254.

13. Choi SE, Rush E, Henry S: Health literacy in Korean immigrants at risk for type 2 diabetes. Journal of immigrant and minority health 2013, 15(3):553-559.

14. Anderson AN, Haardorfer R, Holstad MM, Nguyen MLT, Waldrop-Valverde D: A Path Analysis of Patient and Social-Level Factors on Health Literacy and Retention in Care Among African Americans Living with HIV. AIDS and behavior2020, 24(4):1124-1132.

15. Na S, Ryder AG, Kirmayer LJ: Toward a Culturally Responsive Model of Mental Health Literacy: Facilitating Help-Seeking Among East Asian Immigrants to North 
America. American journal of community psychology 2016, 58(1-2):211-225.

16. Farrell SJ, Dunn M, Huff J, Psychiatric Outreach T, Royal Ottawa Health Care G: Examining Health Literacy Levels in Homeless Persons and Vulnerably Housed Persons with Mental Health Disorders. Community mental health journal 2020, 56(4):645-651.

17. Andargie NB, Jebena MG, Debelew GT: Effectiveness of Checklist-Based Box System Interventions (CBBSI) versus routine care on improving utilization of maternal health services in Northwest Ethiopia: study protocol for a cluster randomized controlled trial. Trials 2020, 21(1):151.

18. Zhu YP, Yue JL, Li WM: Policy participants, policy formulation and improvement of the medical and health conditions of floating population: the path of the policy network. Public Administration Review 2014, 7(04):46-66+183-184.

19. Zhang LY, Cheng XM, Zou ZD, Shen WY, Gao XY: Shanghai Minhang District Foreign Population Health Service Research. China Health Economics 2006(07):45-48.

20. Li CY, Chang C, Ji Y, Shi YH, Wang DX: Survey on the utilization and satisfaction of public health services by young floating population. China Health Education 2012, 28(06):434-437.

21. Chao Y-Y, Seo JY, Katigbak C, Chang Y-P: Utilization of Mental Health Services Among Older Chinese Immigrants in New York City. Community mental health journal 2020:1-13.

22. Alves JFdS, Martins MAC, Borges FT, Silveira C, Muraro AP: Use of health services by Haitian immigrants in Cuiaba-Mato Grosso, Brazil. Ciencia \& saude coletiva 2019, 
24(12):4677-4686.

23. Commission NH: China Floating Population Development Report2018. BeiJing: China population press; 2019.

24. Singh A, Gnanalingham K, Casey A, Crockard A: Quality of Life assessment using the Short Form-12 (SF-12) questionnaire in patients with cervical spondylotic myelopathy - Comparison with SF-36. Spine 2006, 31(6):639-643.

25. Zhang L, Fritzsche K, Liu Y, Wang J, Huang M, Wang Y, Chen L, Luo S, Yu J, Dong Z et al: Validation of the Chinese version of the PHQ-15 in a tertiary hospital. Bmc Psychiatry 2016, 16(1):89-89.

26. Nettelbladt P, Hansson L, Stefansson CG, Borgquist L, Nordstrom G: Test characteristics of the Hopkins Symptom Check List-25 (HSCL-25) in Sweden, using the Present State Examination (PSE-9) as a caseness criterion. Social psychiatry and psychiatric epidemiology 1993, 28(3):130-133.

27. Domanska OM, Firnges C, Bollweg TM, Sorensen K, Holmberg C, Jordan S: Do adolescents understand the items of the European Health Literacy Survey Questionnaire (HLS-EU-Q47) - German version? Findings from cognitive interviews of the project "Measurement of Health Literacy Among Adolescents" (MOHLAA) in Germany. Archives of Public Health 2018, 76(1):46-46.

28. Duong TV, Aringazina A, Baisunova G, Nurjanah, Pham TV, Pham KM, Truong TQ, Nguyen KT, Oo WM, Mohamad E et al: Measuring health literacy in Asia: Validation of the HLS-EU-Q47 survey tool in six Asian countries. Journal of Epidemiology 2017, 27(2):80-86.

29. Xiaoying YLC: Jiangxi Social Science. Jiangxi Social Science 2017, 37(01):229-235. 
30. Xiaoyan YJL: The health awareness and utilization of health services of floating population from the perspective of community_- Research based on the Pearl River Delta. Journal of public management 2014, 11(04):125-135 + 144.

31. Linwe Y: Living and working environment are the key factors affecting the health of migrant workers. Zhejiang Social Sciences 2016(05):75-84 + 157 .

32. commission Nhafp: China Floating Population Development Report 2016. BeiJing: China population press; 2017.

33. Meng X: Does a Different Household Registration Affect Migrants' Access to Basic Public Health Services in China? International Journal of Environmental Research and Public Health 2019, 16(23):4615.

34. Altgeld T: Governance - Government Action: Strengthening the Public's Health by more Public Accountability for Health - Results of the Working Group 11 of the Forum Future Public Health, Berlin 2016. Gesundheitswesen (Bundesverband der Arzte des Offentlichen Gesundheitsdienstes (Germany)) 2017, 79(11):936-939.

35. Thomson K, Hillier-Brown F, Walton N, Bilaj M, Bambra C, Todd A: The effects of community pharmacy-delivered public health interventions on population health and health inequalities: A review of reviews. Preventive Medicine 2019, 124:98-109.

36. Mockford C, Staniszewska S, Griffiths F, Herron-Marx S: The impact of patient and public involvement on UK NHS health care: a systematic review. International Journal for Quality in Health Care 2012, 24(1):28-38.

37. Bavin LM, Owens RG: Complementary Public Service Announcements as a Strategy for Enhancing the Impact of Health-Promoting Messages in Fictional Television Programs. Health communication 2018, 33(5):544-552. 
38. Kobusch $\mathrm{AB}$, Fehr R, Serwe HJ, Protoschill-Krebs G: Evaluation of impact on health--a central responsibility of the public health service. Gesundheitswesen (Bundesverband der Arzte des Offentlichen Gesundheitsdienstes (Germany)) 1995, 57(4):207-213.

39. Davis TC, Arnold C, Berkel H, Nandy I, Jackson RH, Glass J: Knowledge and attitude on screening mammography among low-literate, low-income women. Cancer 1996, 78(9):1912-1920.

40. Wolf MS, Gazmararian JA, Baker DW: Health literacy and functional health status among older adults. Archives of Internal Medicine 2005, 165(17):1946-1952.

41. Williams MV, Baker DW, Parker RM, Nurss JR: Relationship of functional health literacy to patients' knowledge of their chronic disease: A study of patients with hypertension and diabetes. Archives of Internal Medicine 1998, 158(2):166-172.

42. Shun YYZ: Research on the health management problems and countermeasures of floating population in the context of Healthy China. Dongyuelun Cong 2019, 40(6):52-65. 


\section{Figure Legends}

Figure 1. Mediator model of health literacy, health outcomes, and health service utilization 
Table 1. Demographic descriptive statistics $(\mathrm{N}=657)$

\begin{tabular}{|c|c|c|c|c|c|}
\hline & & Frequency & Percentage & Effective & Cumulative \\
\hline & & & & percentage & percentage \\
\hline \multirow{2}{*}{ Gender } & Male & 244 & 37.1 & 37.1 & 37.1 \\
\hline & Female & 413 & 62.9 & 62.9 & 100.0 \\
\hline \multirow{4}{*}{ Age } & 30 years and below & 223 & 33.9 & 33.9 & 33.9 \\
\hline & $31-39$ years & 274 & 41.7 & 41.7 & 75.6 \\
\hline & $40-49$ years & 135 & 20.5 & 20.5 & 96.2 \\
\hline & $>50$ years & 25 & 3.8 & 3.8 & 100.0 \\
\hline \multirow{6}{*}{$\begin{array}{c}\text { Education } \\
\text { level }\end{array}$} & Primary school and below & 39 & 5.9 & 5.9 & 5.9 \\
\hline & Middle school & 317 & 48.2 & 48.2 & 54.2 \\
\hline & Highschool/technical & 158 & 24.0 & 24.0 & 78.2 \\
\hline & secondary school & & & & \\
\hline & Junior college & 81 & 12.3 & 12.3 & 90.6 \\
\hline & Bachelor's degree & 62 & 9.4 & 9.4 & 100.0 \\
\hline
\end{tabular}


Table 2. Reliability coefficient, mean, and standard deviation

\begin{tabular}{|c|c|c|c|c|c|c|}
\hline & $\begin{array}{l}\text { Reliability } \\
\text { coefficient }\end{array}$ & & Minimum & Maximum & Mean & $\begin{array}{l}\text { Standard } \\
\text { deviation }\end{array}$ \\
\hline \multirow{3}{*}{$\begin{array}{l}\text { Health } \\
\text { literacy }\end{array}$} & .939 & Healthcare & 1 & 4 & 2.822 & 0.642 \\
\hline & .916 & Disease prevention & 1 & 4 & 2.952 & 0.590 \\
\hline & .939 & Health promotion & 1 & 4 & 3.035 & 0.608 \\
\hline \multirow{4}{*}{$\begin{array}{c}\text { Health } \\
\text { services }\end{array}$} & .760 & Vaccination services & 1 & 5 & 2.504 & 1.182 \\
\hline & & Health examination & 1 & 5 & 2.763 & 1.374 \\
\hline & & Health education & 1 & 5 & 2.616 & 1.295 \\
\hline & & Health record & 1 & 5 & 2.501 & 1.306 \\
\hline \multirow{3}{*}{$\begin{array}{c}\text { Health } \\
\text { outcome }\end{array}$} & .874 & Self-evaluated health & 1 & 5 & 2.282 & 1.050 \\
\hline & & Physiological health & 1 & 5 & 2.379 & 1.173 \\
\hline & & Psychological health & 1 & 5 & 2.414 & 1.150 \\
\hline
\end{tabular}


Table 3. Model confirmatory factor analysis

\begin{tabular}{lllllll}
\hline Questionnaire & CMIN/DF & NFI & IFI & TLI & CFI & RMSEA \\
\hline Standard level & $<5$ & $>0.8$ & $>0.8$ & $>0.8$ & $>0.8$ & $<0.08$ \\
Good level & $<3$ & $>0.9$ & $>0.9$ & $>0.9$ & $>0.9$ & $<0.05$ \\
Health literacy & 2.99 & 0.844 & 0.89 & 0.885 & 0.89 & 0.055 \\
Health service utilization & 2.577 & 0.992 & 0.995 & 0.986 & 0.995 & 0.049 \\
Health gain & - & - & - & - & - & - \\
\hline
\end{tabular}




\section{Table 4. Variable correlation}

\begin{tabular}{|c|c|c|c|c|c|c|c|c|c|c|c|c|}
\hline & 1 & 2 & 3 & 4 & 5 & 6 & 7 & 8 & 9 & 10 & 11 & 12 \\
\hline Healthcare & 1 & & & & & & & & & & & \\
\hline Disease prevention & $.799^{* *}$ & 1 & & & & & & & & & & \\
\hline Health promotion & $.671^{* *}$ & $.800^{* *}$ & 1 & & & & & & & & & \\
\hline Vaccination services & $.340^{* *}$ & $.369^{* *}$ & $.353^{* *}$ & 1 & & & & & & & & \\
\hline Health examination & $.429^{* *}$ & $.426^{* *}$ & $.411^{* *}$ & $.413^{* *}$ & 1 & & & & & & & \\
\hline Health education & $.391^{* *}$ & $.421^{* *}$ & $.426^{* *}$ & $.411^{* *}$ & $.544^{* *}$ & 1 & & & & & & \\
\hline Health record & $.363^{* *}$ & $.368^{* *}$ & $.402^{* *}$ & $.419^{* *}$ & $.643^{* *}$ & $.605^{* *}$ & 1 & & & & & \\
\hline Self-evaluated health & $.381^{* *}$ & $.359^{* *}$ & $.396^{* *}$ & $.234^{* *}$ & $.278^{* *}$ & $.275^{* *}$ & $.247^{* *}$ & 1 & & & & \\
\hline Physiological health & $.412^{* *}$ & $.400^{* *}$ & $.436^{* *}$ & $.293^{* *}$ & $.356^{* *}$ & $.354^{* *}$ & $.331^{* *}$ & $.697^{* *}$ & 1 & & & \\
\hline Psychological health & $.386^{* *}$ & $.355^{* *}$ & $.388^{* *}$ & $.233^{* *}$ & $.317^{* *}$ & $.348^{* *}$ & $.308^{* *}$ & $.669^{* *}$ & $.757^{* *}$ & 1 & & \\
\hline Health service utilization & $.186^{* *}$ & $.182^{* *}$ & $.175^{* *}$ & $.490^{* *}$ & $.604^{* *}$ & $.581^{* *}$ & $.632^{* *}$ & $.165^{* *}$ & $.280^{* *}$ & $.259^{* *}$ & 1 & \\
\hline Health outcome & $.260^{* *}$ & $.242^{* *}$ & $.219^{* *}$ & $.205^{* *}$ & $.295^{* *}$ & $.269^{* *}$ & $.263^{* *}$ & $.606^{* *}$ & $.691^{* *}$ & $.677^{* *}$ & $.327^{* *}$ & 1 \\
\hline Health literacy & $.904^{* *}$ & $.944^{* *}$ & $.898^{* *}$ & $.387^{* *}$ & $.462^{* *}$ & $.451^{* *}$ & $.412^{* *}$ & $.414^{* *}$ & $.455^{* *}$ & $.412^{* *}$ & $.198^{* *}$ & $.263^{* *}$ \\
\hline
\end{tabular}

$* * \mathrm{p}<0.01(2$-tailed $)$ 
Table 5. Path test

\begin{tabular}{lccccc}
\hline Path & Estimate & S.Estimate & S.E. & C.R. & P \\
\hline Health service utilization<---healthcare & 0.219 & 0.227 & 0.06 & 3.643 & $* * *$ \\
Health service utilization<---disease prevention & 0.118 & 0.112 & 0.079 & 1.493 & 0.135 \\
Health service utilization<---health promotion & 0.305 & 0.299 & 0.065 & $4.704 * * *$ \\
Health outcomes<---health service utilization & 0.459 & 0.29 & 0.085 & $5.428 * * *$ \\
Health outcomes<---healthcare & 0.328 & 0.215 & 0.092 & 3.566 & $* * *$ \\
Health outcomes<---disease prevention & -0.14 & -0.084 & 0.121 & -1.15 & 0.25 \\
Health outcomes<---health promotion & 0.401 & 0.248 & 0.099 & 4.06 & $* * *$ \\
\end{tabular}


Table 6. Mediation effects test

\begin{tabular}{|c|c|c|c|c|c|c|}
\hline Path & Effects & Effect size & S.E. & P-value & $\overline{\text { LLCI }}$ & $\overline{\text { ULCI }}$ \\
\hline Healthcare $\rightarrow$ health service & Total effects & 0.28 & 0.051 & 0.001 & 0.174 & 0.378 \\
\hline utilization $\rightarrow$ health & Direct effect & 0.215 & 0.052 & 0.001 & 0.108 & 0.313 \\
\hline outcomes & Mediating effects & 0.066 & 0.022 & 0.001 & 0.023 & 0.299 \\
\hline Disease prevention $\rightarrow$ health & Total effects & -0.051 & 0.08 & 0.112 & -0.206 & 0.112 \\
\hline service utilization $\rightarrow$ health & Direct effect & -0.084 & 0.08 & 0.32 & -0.236 & 0.078 \\
\hline outcomes & Mediating effects & 0.033 & 0.021 & 0.112 & -0.007 & 0.075 \\
\hline Health promotion $\rightarrow$ health & Total effects & 0.335 & 0.07 & 0.001 & 0.192 & 0.467 \\
\hline service utilization $\rightarrow$ health & Direct effect & 0.248 & 0.072 & 0.001 & 0.101 & 0.389 \\
\hline outcomes & Mediating effects & 0.087 & 0.022 & 0.001 & 0.047 & 0.133 \\
\hline
\end{tabular}




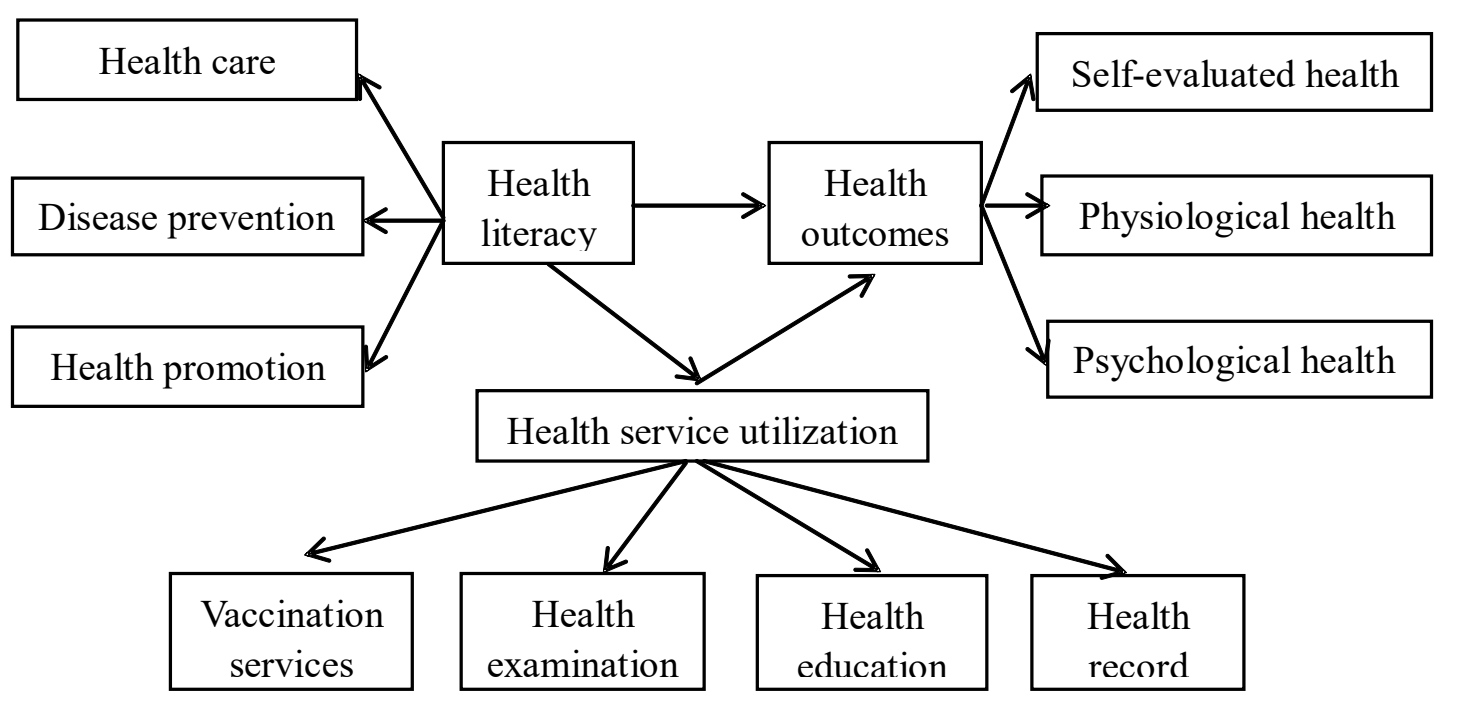

Fig. 1 
Figures

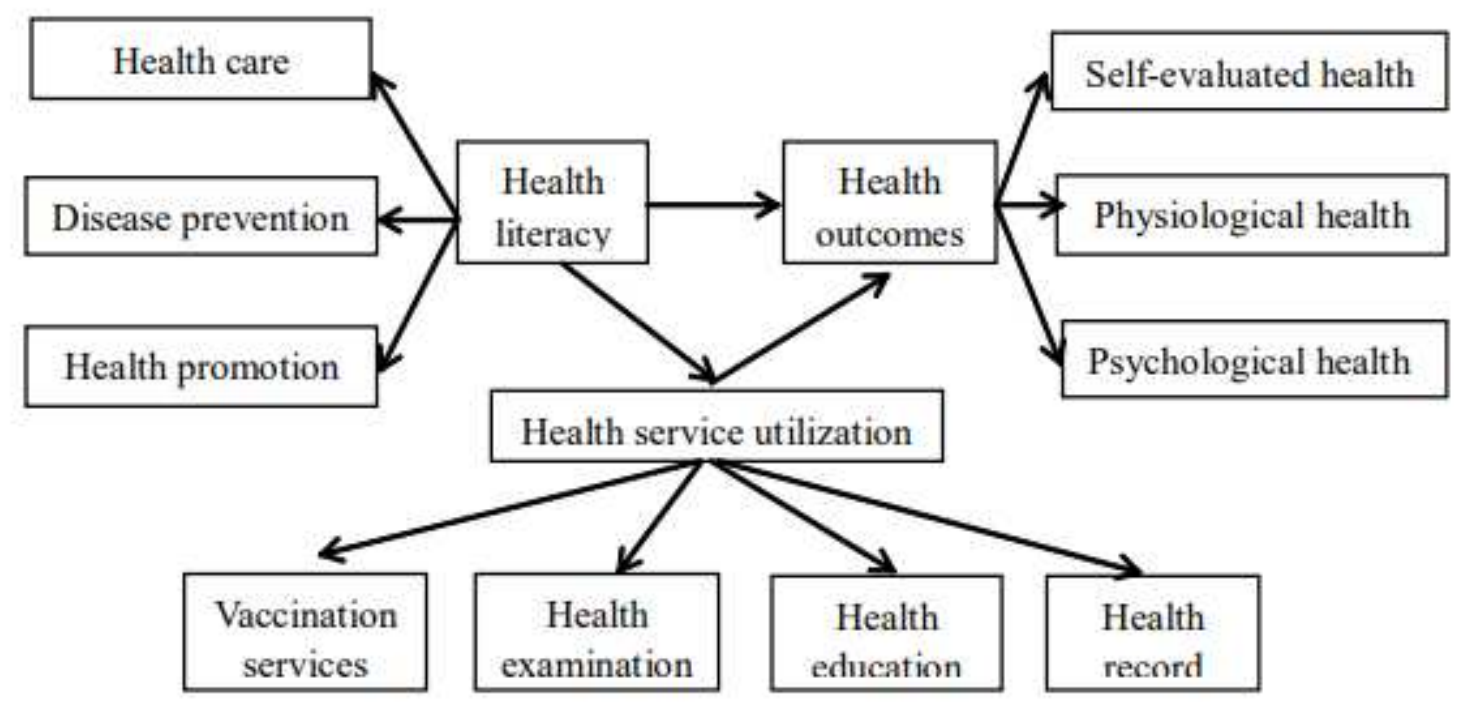

Figure 1

Mediator model of health literacy, health outcomes, and health service utilization 